\title{
Cryogenic Thermal Distortion Model Validation for the JWST ISIM Structure
}

\author{
John Johnston*a and Emmanuel Cofie ${ }^{\mathrm{b}}$ \\ ${ }^{a}$ NASA Goddard Space Flight Center, Greenbelt, MD, USA 20711 \\ ${ }^{\mathrm{b}} \mathrm{SGT}$, Inc., Seabrook, MD, 20706
}

\begin{abstract}
The James Webb Space Telescope (JWST) Integrated Science Instrument Module (ISIM) Structure is a precision optical metering structure for the JWST science instruments. Optomechanical performance requirements place stringent limits on the allowable thermal distortion of the metering structure. A significant effort was completed to develop capabilities to predict and metrologize cryogenic thermal distortion of the ISIM Structure. This paper focuses on thermal distortion finite element modeling, analysis, and model validation. Extensive thermal distortion analysis was completed during the design phase for the ISIM Structure to demonstrate that thermal distortion requirements were achieved. Comparison of measurements from recently completed cryogenic testing and model predictions demonstrate the adequacy of thermal distortion modeling uncertainty factors adopted during the design phase, and provide bounds on the accuracy of the model predictions. This paper will provide an overview of the test configurations, describe the thermal distortion models of the tests, and provide a comparison of test results and analytical predictions from the models.
\end{abstract}

Keywords: JWST, ISIM Structure, Thermal Distortion, Finite Element Analysis, Model Validation

\section{INTRODUCTION}

\subsection{Overview of JWST, ISIM, and the ISIM Structure}

The James Webb Space Telescope (JWST) is a large, infrared space telescope consisting of an optical telescope element (OTE), an integrated science instrument module (ISIM), a spacecraft, and a sunshield (Figure 1). ${ }^{1}$ ISIM consists of the JWST science instruments (NIRCam, MIRI, NIRSpec), a fine guidance sensor (FGS), the ISIM Structure, and thermal and electrical subsystems (Figure 1). ${ }^{2}$ The ISIM Structure is an approximately $2 \mathrm{~m} \mathrm{x} 2 \mathrm{~m} \times 1.5 \mathrm{~m}$ bonded composite frame that acts as a metering structure between the instruments/guider and the telescope. ${ }^{3,4}$ The ISIM Structure interfaces to the OTE via a kinematic mount (KM) system consisting of two bipods and two monopods. The ISIM Structure and the supported instruments are designed to operate at cryogenic temperatures $(\sim 35 \mathrm{~K})$, and thermal distortion performance is critical to maintaining the alignment of the instruments to the ISIM Structure. Significant effort has been expended on the development of capabilities to predict and measure cryogenic thermal distortion in order to ensure that the requirements are met and to provide validated models for prediction of on-orbit performance.
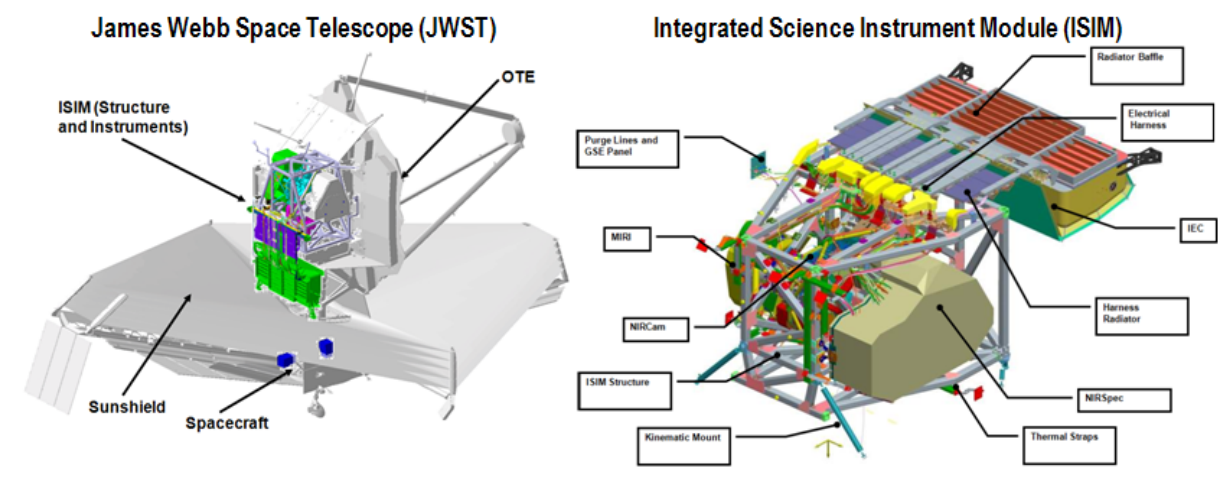

Figure 1. James Webb Space Telescope (JWST) and Integrated Science Instrument Module (ISIM).

*John.D.Johnston@nasa.gov; phone 301-286-2836; fax 301-286-2696; www.nasa.gov, www.jwst.nasa.gov. 


\subsection{ISIM Structure Thermal Distortion Tests}

ISIM Structure development and verification followed a building blocks approach starting with the development and testing of material samples, followed by bonded joint and subassembly level activities, and culminating with the structural verification program for the flight hardware. A summary of the initial "building block" tests and corresponding model validation for the ISIM Structure is provided in Reference 5. Prior to testing the protoflight ISIM Structure, a cryogenic thermal performance test, the Chamber Certification Test, was completed for the mechanical ground support equipment (MGSE) used to test the flight hardware. Two major cryogenic environmental tests were then completed as part of protoflight ISIM Structure verification program. The Cryoset Test was completed in May 2010 for thermal distortion performance characterization, and the Cryoproof Test was completed in October 2010 for demonstration of cryogenic strength. During each of these tests, the hardware under test was thermal cycled between ambient and cryogenic temperatures with metrology performed via photogrammetry at the warm and cold states. The test was completed in the NASA Goddard Space Environment Simulator (SES) thermal vacuum chamber, which has been outfitted with a new Helium shroud to achieve the required cryogenic temperature test environment. A photogrammetry system was used for metrology during each of the cryogenic tests. Details of the photogrammetry system, its error budgets, and performance will not be discussed in detail here as they have been previously reported. ${ }^{6,7}$

\section{Chamber Certification Test}

The ISIM Chamber Certification Test was completed to check-out the MGSE and metrology system at cryogenic temperatures prior to integration and testing with the flight ISIM Structure. The major items included in the test configuration consisted of the Helium Shroud, photogrammetry system, Lower GESHA which interfaces to the chamber floor, Upper GESHA, and ISIM Test Platform (ITP) which interfaces to the flight ISIM Structure. The most critical MGSE hardware from a distortion standpoint is the ISIM Test Platform (ITP) which acts as a surrogate for the flight telescope interface during ISIM Element level testing.

\section{Cryoset Test}

The ISIM Structure Cryoset Test was completed to verify the thermal distortion performance of the ISIM Structure and two pieces of optical grounds support equipment (OGSE) needed for future ISIM Element Cryovac Tests: the ISIM Alignment Target Fixture, IATF and the Master Alignment Target Fixture (MATF). The test configuration for the Cryoset Test, Figure 2, consists of the ISIM Chamber Certification Test configuration with the addition of the bare flight ISIM Structure, IATF, and MATF. During the Cryoset test, the science instrument interfaces were characterized via metrology tooling called SEPs (Science Instrument Interface Plate Extender Plates). The SEPs provide an array of photogrammetry targets to enable characterization of both translations and rotations of the interfaces.

\section{Cryoproof Test}

The ISIM Structure Cryoproof Test was completed to verify the cryogenic strength of the ISIM Structure. The test configuration for the Cryoset Test, Figure 2, consists of the ISIM Chamber Certification Test configuration with the addition of mass loaded flight ISIM Structure and the IATF.

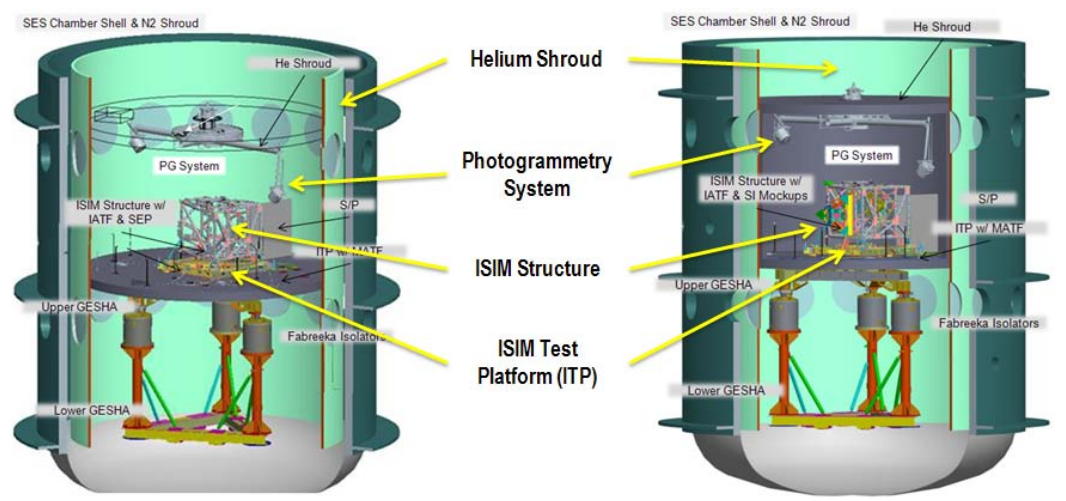

Figure 2. Test configurations for ISIM Structure Cryoset Test and ISIM Structure Cryoproof Test. The test configuration for Chamber Certification test is similar to other tests, but with ISIM Structure removed. 


\subsection{Overview of Paper}

The remainder of this paper will discuss the modeling and analysis methodology utilized to predict the thermal distortion performance of the ISIM Structure and related cryogenic test MGSE. Model validation studies whereby analytical predictions are compared with test measurements will be described. Results from model validation from the ISIM Chamber Certification, ISIM Structure Cryoset, and ISIM Structure Cryoproof tests will be presented.

\section{THERMAL DISTORTION MODELING AND ANALYSIS}

Thermal distortion analysis was completed using NASTRAN finite element analysis software. Detailed structural models were completed for both the flight hardware and associated mechanical ground support equipment in the test setup. Figures 3-5 show the ISIM Chamber Certification Test, ISIM Structure Cryoset, and ISIM Structure Cryoproof test structural models, respectively. The ISIM Structure models used for thermal distortion analysis are high fidelity ( $>2$ million degrees of freedom) structural models. The ISIM Structure composite frame is modeled using solid elements with sufficient fidelity to model details such as bond lines and shapes. Mesh convergence studies were completed to define the mesh size for the global thermal distortion model based on comparison of predictions between high fidelity models of representative bonded joints that served as the reference standard against which successive thermal distortion model mesh sizes could be compared. Material properties used in the models are all tied to materials data specifically generated for the program, in particular temperature-dependent CTE and stiffness properties. The MGSE included in the test configuration models includes all of the hardware in the structural load path from the chamber floor interface up to the flight hardware. The MGSE structural models were of sufficient fidelity to predict the influence of the supporting hardware on the flight structure. Model validation was completed for the ISIM Test Platform which directly interfaces to the flight hardware to confirm the adequacy of this model.

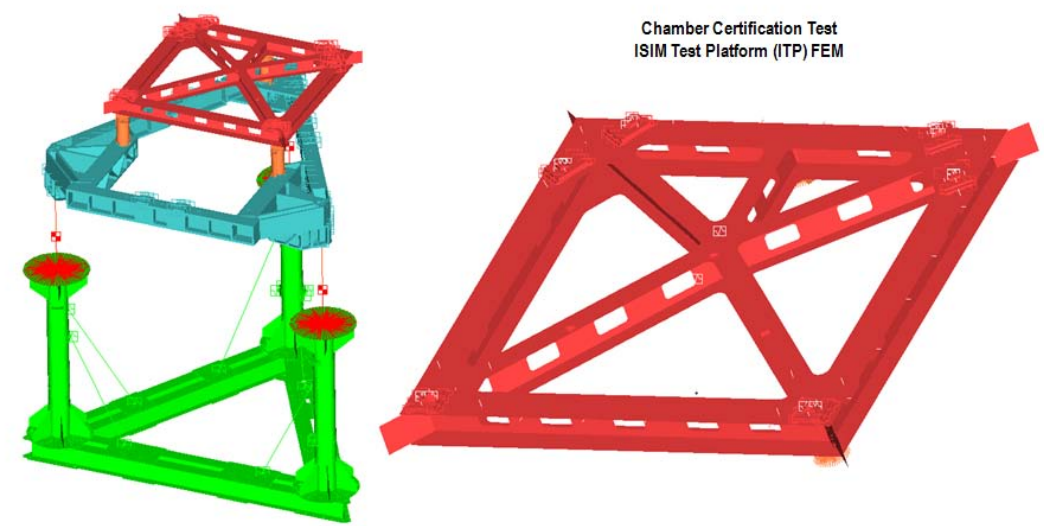

Figure 3. Structural model configuration for ISIM Chamber Certification Test with close-up of ITP shown.

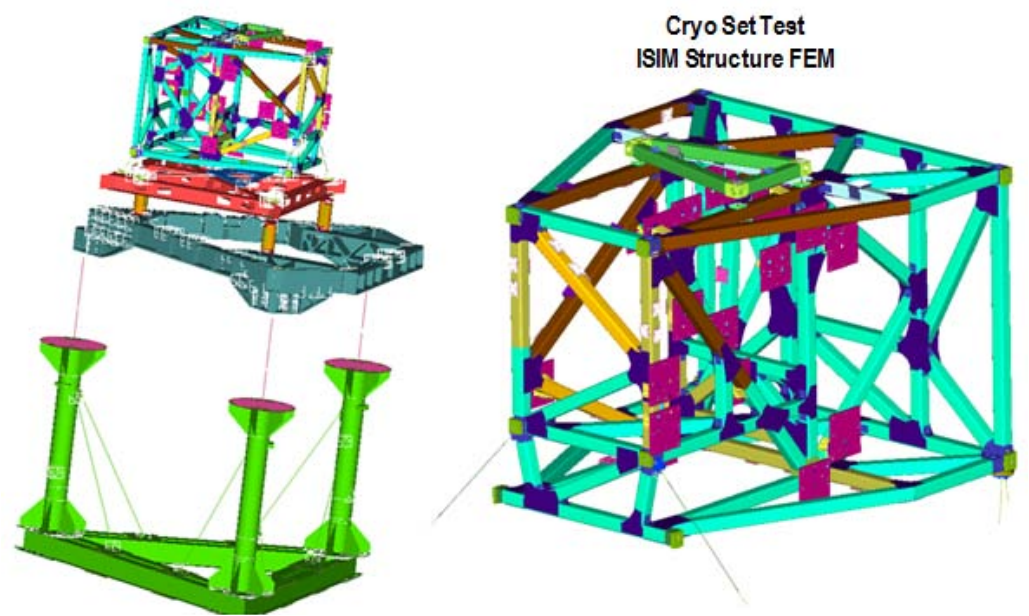

Figure 4. Structural model configuration for ISIM Structure Cryoset Test with close-up of "bare” ISIM Structure shown. 

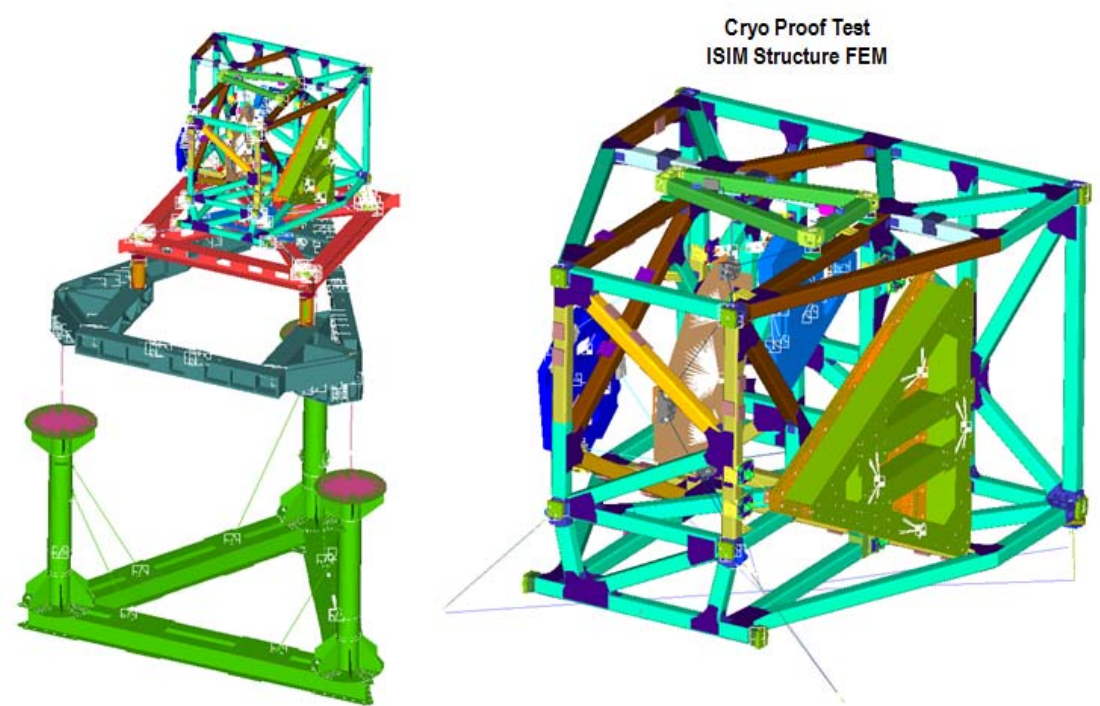

Figure 5. Structural model configuration for ISIM Structure Cryoproof Test with close-up of mass loaded ISIM Structure shown.

The basic thermal distortion analysis completed for each test configuration consists of two steps: (1) temperature mapping and (2) distortion analysis. During the temperature mapping step, temperature sensor measurements are used to generate a temperature value for each node in the flight hardware and MGSE structural models. First, temperature sensor locations were mapped to (associated with) the closest structural model nodes, and the temperatures of mapped nodes in the structural model are set equal to temperature sensor data. Next, a NASTRAN steady state heat analysis is performed to fully "seed" all nodes in the structural model with temperatures. Temperature maps were created for each state at which metrology data was taken, at both ambient and cryogenic temperatures. Figures 6-7 show the temperature distributions in the overall test setup and the ISIM Structure test article at cryogenic operating temperature from the Cryoset and Cryoproof Tests. During the distortion analysis step, the complete temperature map is applied as a thermal load and distortions of the system are calculated via linear static analysis.
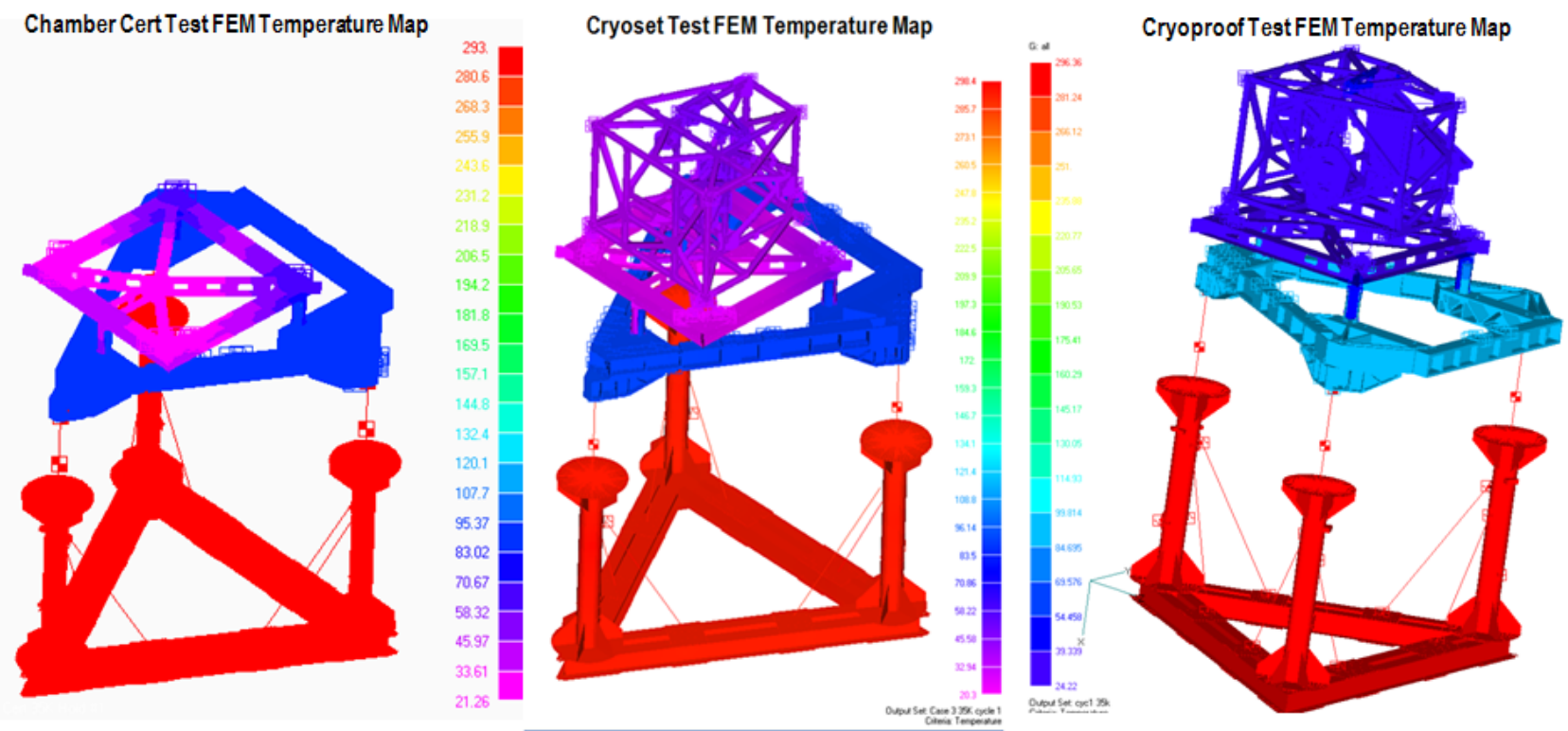

Figure 6. Temperature maps for full test configurations at cryogenic temperature. 

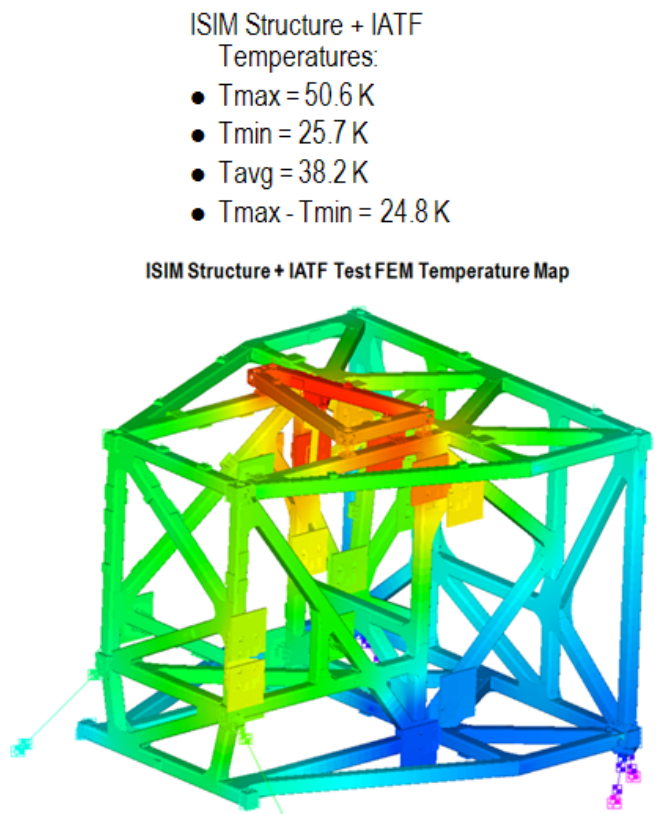

Figure 7. Temperature maps for ISIM Structure at cryogenic temperature.
ISIM Structure + IATF + Mass Sim

Temperatures:

- $T \max =45.1 \mathrm{~K}$

- $\operatorname{Tmin}=25.6 \mathrm{~K}$

- $\mathrm{Tavg}=35.4 \mathrm{~K}$

- $T \max -\mathrm{Tmin}=19.5 \mathrm{~K}$

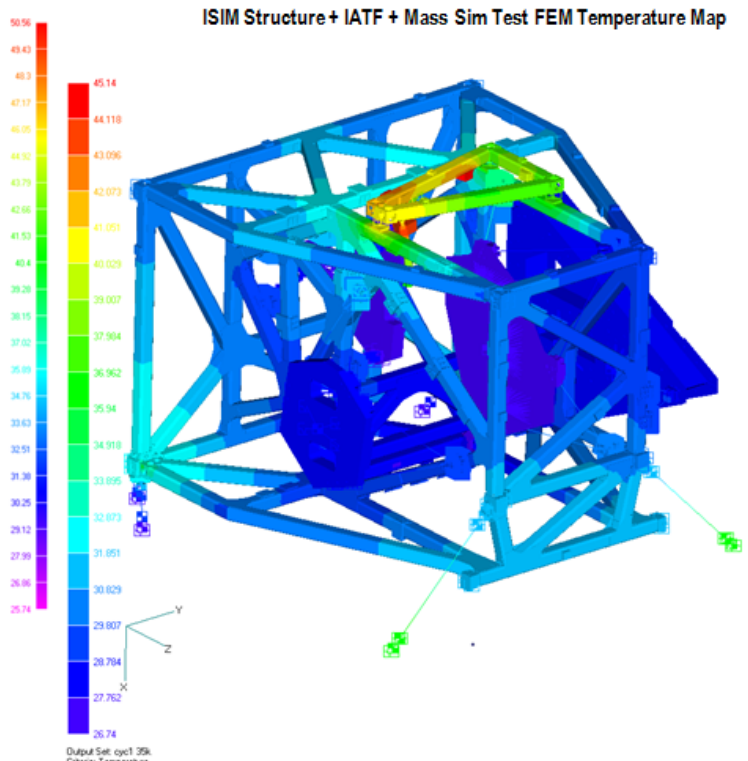

Two types of analysis are used to predict ISIM Structure thermal distortion: nominal analysis and stochastic analysis. Nominal analysis is completed with nominal material properties and geometry in the model. Stochastic analysis is used to predict the uncertainties due to factors such as material property and geometric variability, and provides a mean prediction and a 95\% confidence interval. Modeling uncertainty factors (MUFs) are used to provide conservatism and margin in the thermal distortion predictions. Project guidelines specify MUFs based on the maturity of the hardware design and level of model validation completed. The analysis results presented here include a MUF of 1.6 for nominal model predictions and a MUF of 1.4 on the $95 \%$ confidence interval for stochastic model predictions. Model validation criteria are specified for the two analysis types based and the associated modeling uncertainty factors. The model validation criteria for nominal model predictions is that predictions multiplied by the 1.6 MUF should bound test measurements including measurement error. The model validation criteria for stochastic model predictions is that the predicted uncertainty bandwidth should envelop test measurements including measurement error. The following sections will describe the model validation results for the ISIM Chamber Certification, ISIM Structure Cryoset, and ISIM Structure Cryoproof tests.

\section{CHAMBER CERTIFICATION TEST MODEL VALIDATION}

For the ISIM Chamber Certification Test, model validation was completed for the ISIM Test Platform (ITP). Ambient to cryo coolown motions for the 18 Reference A targets (associated with the ISIM kinematic mount interfaces) with respect to Reference A were compared between analysis and test. The magnitude of the measured and predicted V1 translations (out-of-plane for the ITP) is less than PG warm-to-cold measurement uncertainty of 32 microns, so no model validation is completed for distortions in that direction. Figure 8 presents a comparison of the motions of the 18 targets in the V2 and V3 (in-plane) directions. The nominal/mean motions show excellent agreement with translation measurements agreeing to within 14 microns. Nominal predictions with a modeling uncertainty factor of 1.6 bound the measured performance. Stochastic model predictions including 2-sigma uncertainty bandwidth with modeling uncertainty factor of 1.4 envelop measured performance. Additionally, the ambient to average cryogenic temperature average cooldown strains throughout the ITP (all invar construction) show excellent agreement between the all-up test, analysis, and 
coupon level testing of the invar lot used to fabricate the hardware: -361 ppm from all-up test, -366 from the system FEM, and -361 from coupon testing. Subsequent thermal distortion characterization of the ITP during the ISIM Structure Cryoset and Cryoproof tests demonstrated that the ITP cooldown behavior is unchanged between tests (maximum differences less than photogrammetry measurement uncertainty).

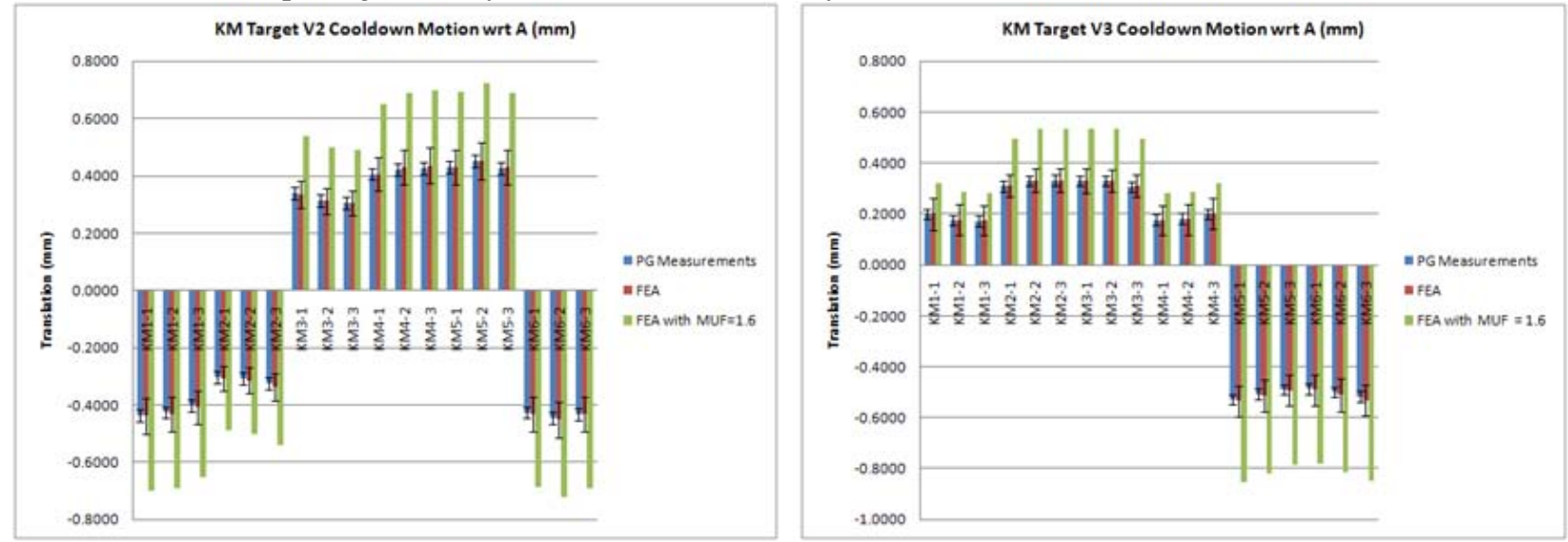

Figure 8. Comparison of ITP target translations with respect to Reference A from ISIM Chamber Certification Test.

\section{CRYOSET TEST MODEL VALIDATION}

For the ISIM Structure Cryoset Test, model validation was completed for the protoflight ISIM Structure, ISIM Test Platform (ITP), Master Alignment Target Fixture (MATF), and ISIM Alignment Target Fixture (IATF). Results for the ISIM Structure will be presented here. Comparisons of measurements and predictions for the MATF (all invar) are similar to the ITP, and comparisons for the IATF (composite bonded structure) show similar results to the ISIM Structure. Additionally, ITP performance did not change compared to the Chamber Certification test. For the ISIM Structure, ambient to cryo (cycle 1 operational temperature) motions for the ISIM BCG-to-Reference ACG, SI Pads-toReference BCG, and ISIM Structure general targets-to-Reference BCG were compared between analysis and test.

Figure 9 presents results for cooldown motions of Reference BCG with respect to Reference ACG which describes rigid body motion of the ISIM Structure on its kinematic mounts. Translations are primarily in the V1 direction due to axial cooldown distortion of the kinematic mount struts. Rotations are primarily about the V2 axis due in large part to differences in axial length change of the bipod and monopod mount struts. The nominal/mean V1 translation for BCGto-ACG shows excellent agreement with translation measurements agreeing to within 45 microns out of a total motion of 421 microns or about 10\% difference. The nominal/mean R2 rotation for BCG-to-ACG also shows excellent agreement with rotation measurements agreeing to 0.1 arcmin out of a total rotation of 1.1 arcmin or again about $10 \%$ difference. Nominal predictions with a modeling uncertainty factor of 1.6 bound the measured performance. Stochastic model predictions including 2-sigma uncertainty bandwidth with modeling uncertainty factor of 1.4 envelop measured performance.
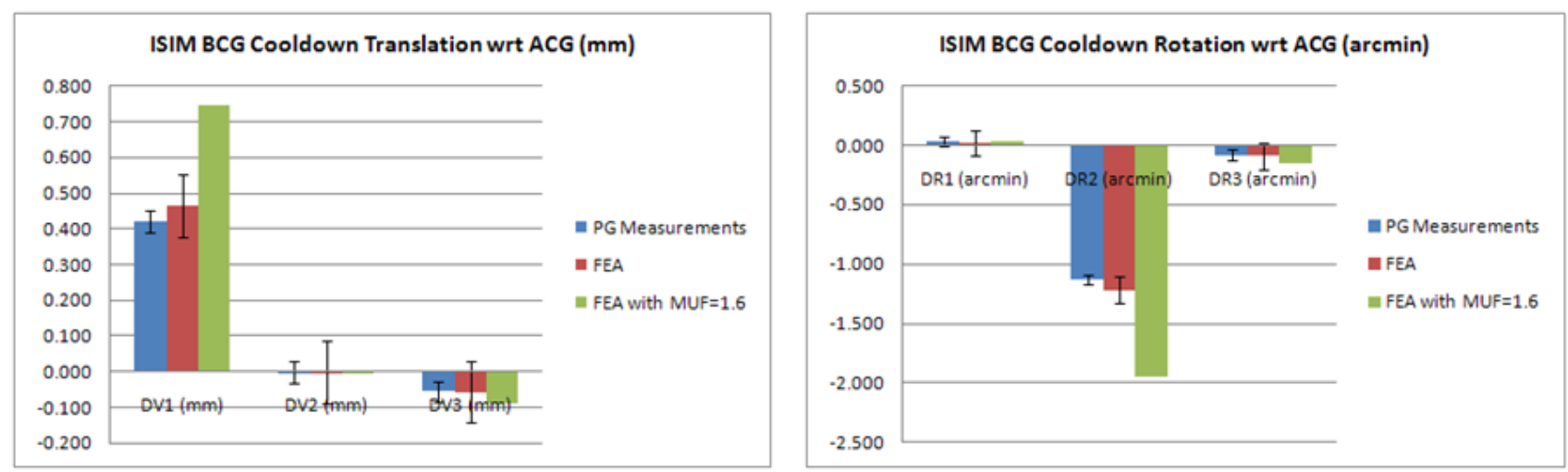

Figure 9. Comparison of ISIM Structure BCG-to-ACG motions in six degrees of freedom from ISIM Structure Cryoset Test. 
Figure 10 presents representative results for cooldown translations of the science instrument interfaces with respect to Reference BCG. This performance metric relates to internal distortion of the ISIM Structure. Maximum cooldown translations with respect to BCG are on the order of 200 microns, and significant motions were defined as translations greater than 50 microns (the 3-sigma photogrammetry error bar). For significant translations, the nominal model predictions without modeling uncertainty factor agree with test measurements to within 50 microns. Figure 10 compares the predicted and measured values for the cooldown translations for the NIRSpec science instrument interfaces with respect to the ISIM BCG Reference.

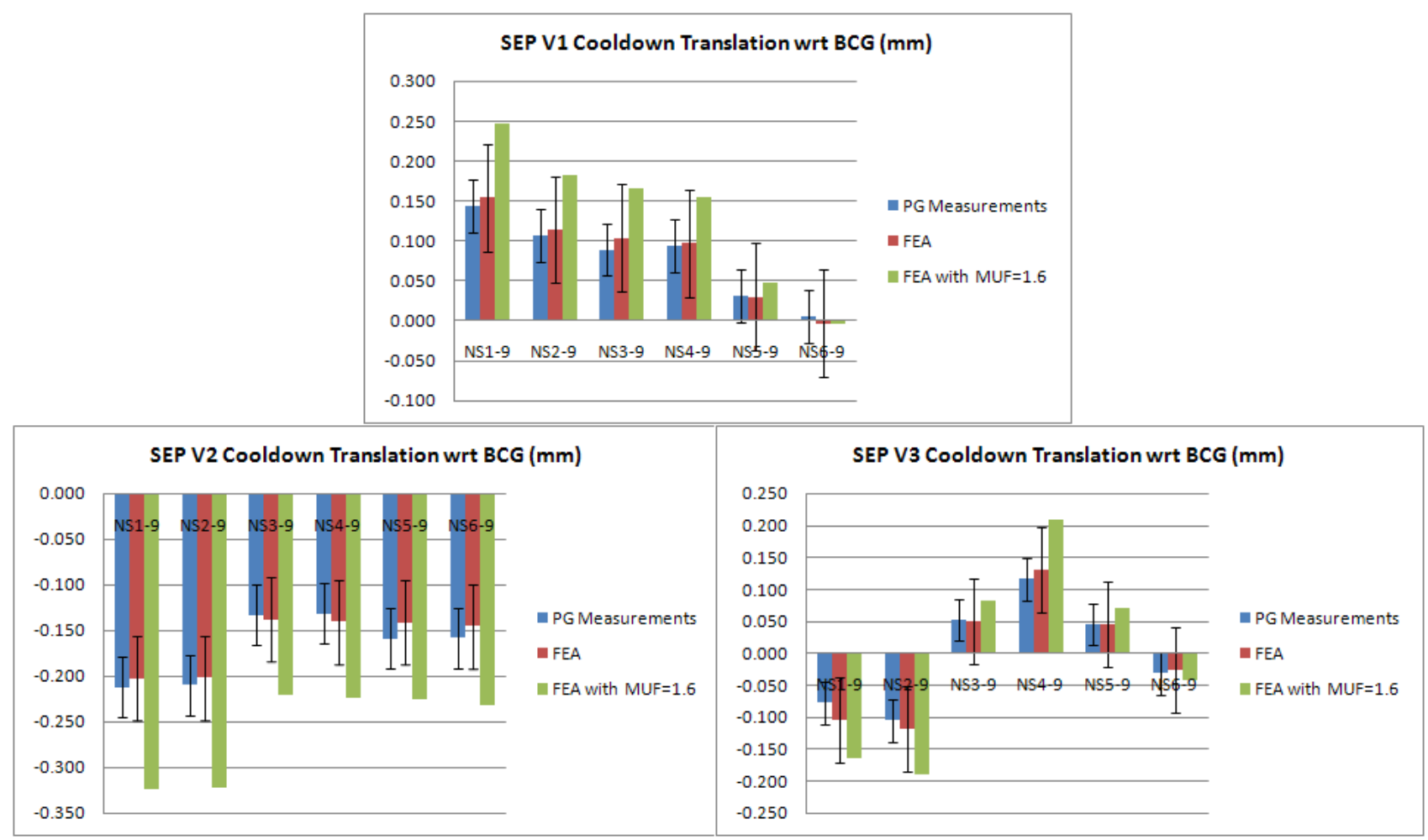

Figure 10. Comparison of ISIM Structure SI Pads to BCG for NIRSpec interface translations with respect to Reference BCG from ISIM Structure Cryoset Test.

Figure 11 presents representative results for cooldown rotations of the science instrument interfaces with respect to Reference BCG, a performance metric related to internal distortion of the ISIM Structure. Maximum cooldown rotations with respect to BCG are on the order of 1.3 arcmin, and significant rotations were defined as those greater than 0.7 arcmin (the 3-sigma photogrammetry error bar). The measured rotations were overall small compared to performance requirements ( $<5$ arcmin required) and only 11 SEP rotation DOFs (out of 57) showed cooldown rotations greater than the 0.71 arcmin validation threshold. For significant rotations, the nominal model predictions without modeling uncertainty factor agree with test measurements to within 0.6 arcmin (about the PG measurement error). Model validation for rotations was challenging due to the small magnitude of the measured rotations compared to measurement uncertainties, and the model validation criteria were only partially satisfied for these degrees of freedom. In some cases, nominal model predictions including a modeling uncertainty factor of 1.6 bounded the test measurements. In all cases, stochastic model predictions including an uncertainty bandwidth based on a modeling uncertainty factor of 1.4 multiplied by the 2-sigma uncertainty bandwidth associated with material and geometric uncertainties envelope or overlap with the test measurements. Thus, the nominal model is considered partially validated and the stochastic model can be considered fully validated for the SI pad rotation degrees of freedom. 


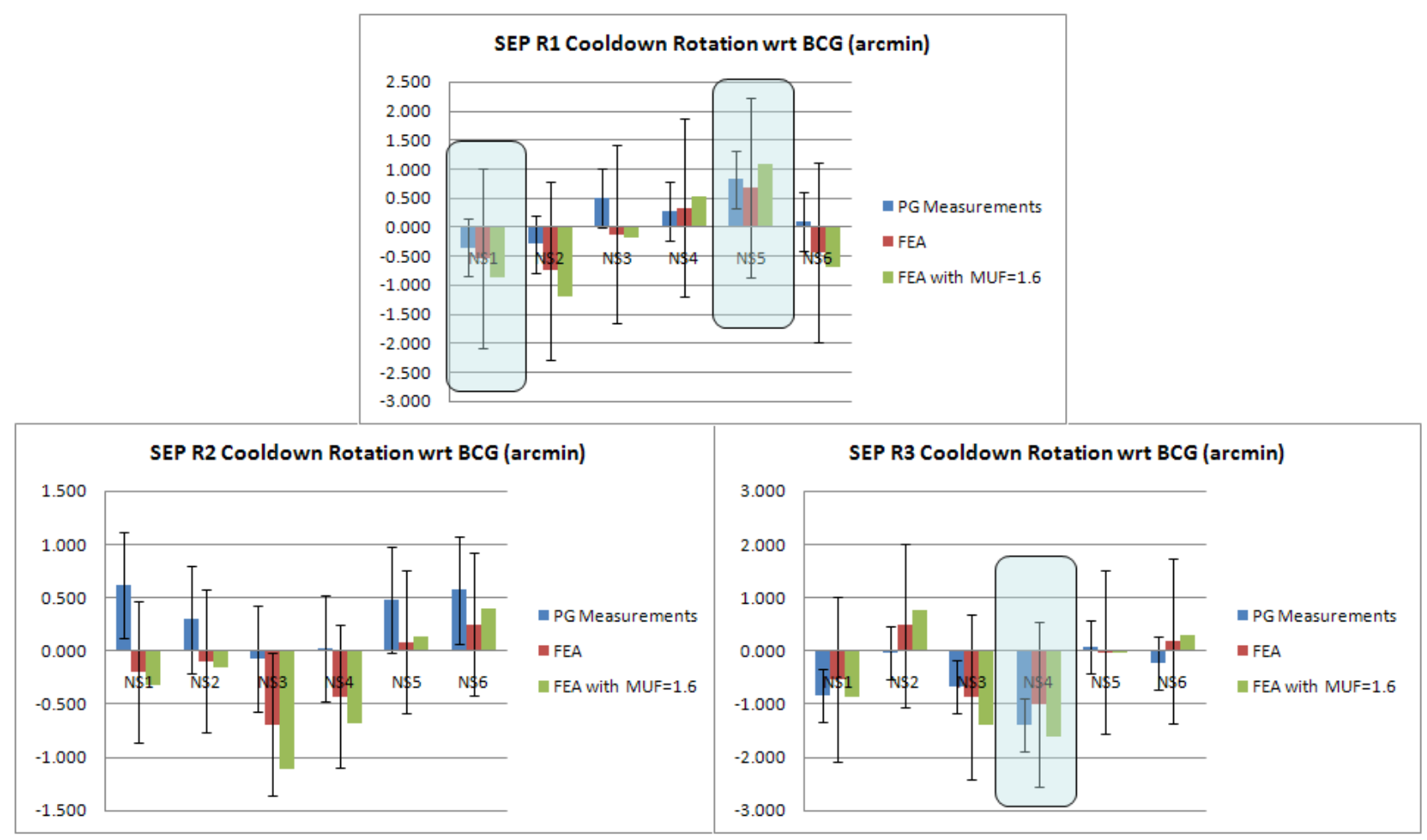

Figure 11. Comparison of ISIM Structure SI Pads to BCG for NIRSpec interface rotations with respect to Reference BCG from ISIM Structure Cryoset Test. Significant rotations greater than the 0.7 arcmin 3-sigma PG uncertainty are highlighted with boxes (3 instances).

\section{CRYOPROOF TEST MODEL VALIDATION}

For the ISIM Structure Cryoproof Test, model validation was completed for the protoflight ISIM Structure, ISIM Test Platform (ITP), and ISIM Alignment Target Fixture (IATF). Results for the ISIM Structure will be presented here. As with the Cryoset test, comparisons for the IATF (composite bonded structure) show similar results to the ISIM Structure, and performance of the ITP was unchanged compared to previous tests. For the ISIM Structure, ambient to cryo cooldown motions for the ISIM BCG-to-Reference ACG and ISIM Structure general targets-to-Reference BCG were compared between analysis and test. Science instrument interfaces could not be metrologized during the Cryoproof test due to the presence of the science instrument mass simulators. Figure 12 presents results for cooldown motions of Reference BCG with respect to Reference ACG. The test measurements and predictions are similar to the ISIM Structure Cryoset Test. Once again, the nominal/mean V1 translation for BCG-to-ACG shows excellent agreement with translation measurements agreeing to within 18 microns out of a total motion of 435 microns or about $5 \%$ difference. The nominal/mean R2 rotation for BCG-to-ACG also shows excellent agreement with rotation measurements agreeing to 0.1 arcmin out of a total rotation of 1.1 arcmin or about $10 \%$ difference. Model validation criteria are satisfied for both the nominal and stochastic analyses. 

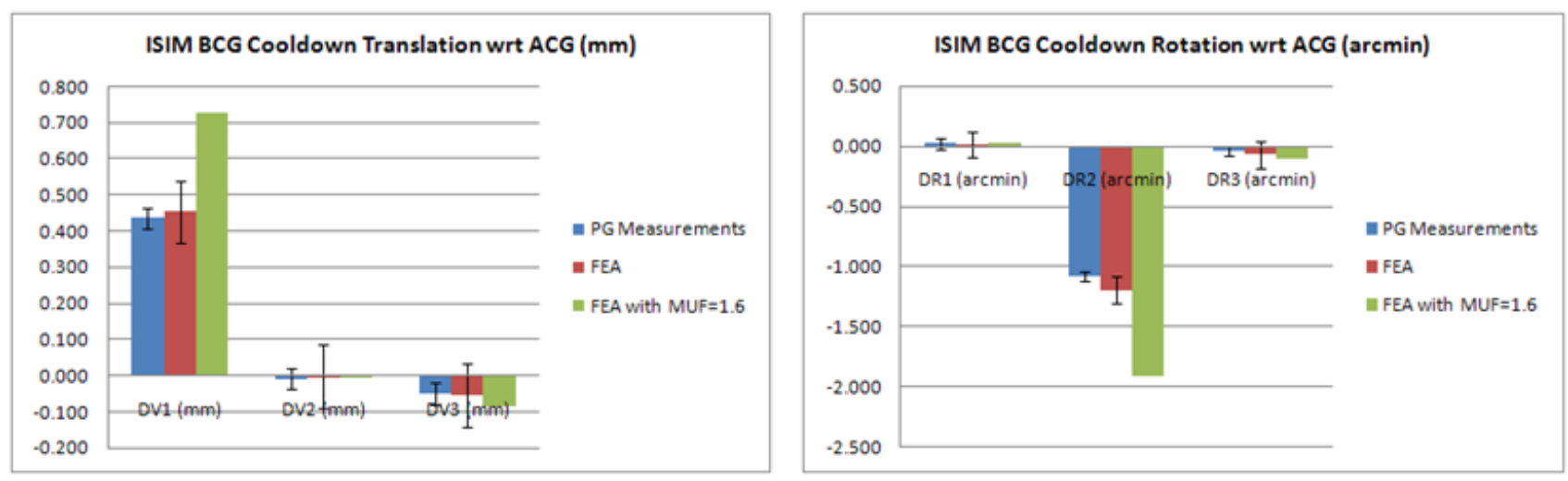

Figure 12. Comparison of ISIM Structure BCG-to-ACG motions in six degrees of freedom from ISIM Structure Cryoproof Test.

\section{COMPARISON OF CRYOSET AND CRYOPROOF TESTS}

A comparison was made between the ISIM Structure cooldown performance measured and predicted in the bare (during the Cryoset Test) and mass-loaded (during the Cryoproof Test) configurations. The metrics selected were: BCG-toACG motion indicative of rigid body motion of the ISIM Structure on its kinematic mounts and ITOR CG-to-BCG motion indicative of general target motion on the "top" (-V1) deck of the ISIM Structure relative to Reference BCG. Table 1 presents a comparison of test results and analytical predictions from the nominal analysis with no MUF for both test configurations. Photogrammetry measurements greater than the 56 micron 3-sigma validation threshold for significant motions are highlighted in the table. The differences in measured and predicted cooldown performance between the two tests are less than 14 microns for both metrics. This demonstrates that the influence of differences in thermal environment on the order of $<10 \mathrm{~K}$ and the presence of mass loading did not appreciably change thermal distortion performance as predicted.

Table 1. Comparison of cooldown translation measurements and predictions from ISIM Structure Cryoset and Cryoproof Tests.

\begin{tabular}{|c|c|c|c|c|c|c|c|c|c|c|c|}
\hline \multirow[b]{2}{*}{ Test } & \multirow{2}{*}{$\begin{array}{c}\text { Target } \\
\text { Centroid }\end{array}$} & \multirow[b]{2}{*}{ Reference } & \multicolumn{3}{|c|}{ PG Measurements } & \multicolumn{3}{|c|}{ FEA with No MUF } & \multicolumn{3}{|c|}{ Difference (PG - FEA) } \\
\hline & & & $\Delta \mathrm{V} 1(\mathrm{~mm})$ & $\Delta \mathrm{V} 2$ (mm) & $\Delta \mathrm{V} 3(\mathrm{~mm})$ & $\Delta \mathrm{V1}(\mathrm{mm})$ & $\Delta \mathrm{V} 2(\mathrm{~mm})$ & $\Delta \mathrm{V} 3(\mathbf{m m})$ & $\Delta \mathrm{V} 1$ (mm) & $\Delta \mathrm{V} 2$ (mm) & $\Delta \mathrm{V} 3(\mathbf{m m})$ \\
\hline Cryoset & BCG & ACG & 0.421 & -0.001 & -0.055 & 0.466 & -0.001 & -0.057 & -0.045 & 0.000 & 0.002 \\
\hline Cryoproof & $\mathrm{BCG}$ & ACG & 0.435 & -0.008 & -0.050 & 0.454 & -0.001 & -0.053 & -0.018 & -0.007 & 0.003 \\
\hline Difference (CS-CP) & $B C G$ & ACG & -0.014 & 0.007 & -0.005 & 0.013 & 0.000 & -0.004 & & & \\
\hline Cryoset & ITOR CG & BCG & 0.142 & 0.057 & 0.097 & 0.129 & 0.055 & 0.114 & 0.013 & 0.002 & -0.017 \\
\hline Cryoproof & ITOR CG & BCG & 0.147 & 0.058 & 0.085 & 0.127 & 0.054 & 0.111 & 0.020 & 0.003 & -0.027 \\
\hline Difference (CS-CP) & ITOR CG & BCG & -0.006 & 0.000 & 0.012 & 0.002 & 0.001 & 0.003 & & & \\
\hline
\end{tabular}

\section{SUMMARY}

Cryogenic testing and associated thermal distortion model validation for the JWST ISIM Structure were successfully completed in 2010. Detailed comparisons were made between test measurements and analytical predictions from nominal and stochastic analyses for the cooldown performance of the protoflight ISIM Structure and critical MGSE. Results from these comparisons demonstrate that the models accurately predict thermal distortion performance and meet model validation goals tied to the type of analysis and associated model uncertainty factors. The model validation studies established typical bounds on the accuracy of predictions of better than 15 microns for the metallic MGSE (ITP) and better than 50 microns for the composite flight structure. The validated models will be used for the analysis of both future test configurations and on-orbit performance. Future cryogenic thermal vacuum testing of the integrated ISIM Element, consisting of the science instruments and associated subsystems mounted to the ISIM Structure, will build on the success of the ISIM Structure cryogenic testing and associated model validation to verify integrated ISIM performance requirements. 


\section{REFERENCES}

[1] Sabelhaus, P.A., Campbell, D., Clampin, M., Decker, J., Greenhouse, M., Johns, A., Menzel, M., Smith, R., Sullivan, P., “An overview of the James Webb Space Telescope (JWST) project,” Proc. SPIE Vol. 5899, p. 241-254, UV/Optical/IR Space Telescopes: Innovative Technologies and Concepts II, (2005).

[2] Bos, B.J., Davila, P.S., Jurotich, M., Hobbs, G., Lightsey, P.A., Contreras, J., Whitman, T., “The James Webb Space Telescope instrument suite layout: optical system engineering considerations for a large deployable space telescope,” Proc. SPIE Vol. 5487, p. 734-745, Optical, Infrared, and Millimeter Space Telescopes, (2004).

[3] Kunt, C., Johnston, J.D., Bartoszyk, A., Hendricks, S., "Development and sizing of the JWST Integrated Science Instrument Module (ISIM) metering structure,” Proc. SPIE Vol. 6273, Optomechanical Technologies for Astronomy, (2006).

[4] Bartoszyk, A., Johnston, J.D., Kaprielian, C., Kuhn, J. Kunt, C., Rodini, B., Young, D., "Design/analysis of the JWST ISIM bonded joints for survivability at cryogenic temperatures,” Proc. SPIE Vol. 5868, p. 171-180, Optical Materials and Structures Technologies II, (2005).

[5] Johnston, J.D., Cofie, E., "An Overview of Thermal Distortion Modeling, Analysis, and Model Validation for the JWST ISIM Structure,” AIAA Structures, Structural Dynamics, and Materials Conference, Denver, CO, AIAA2011-2161, (2011).

[6] Nowak, M.D., Crane, J.A., Davila, P.S., Hylan, J.E., Johnston, J.D., Ohl, R.G., et al., “Cryogenic performance of a high precision photogrammetry system for verification of the James Webb Space Telescope Integrated Science Instrument Module and associated ground support equipment structural alignment requirements,” Proc. SPIE 7793, Optical System Alignment, Tolerancing, and Verification IV, (2010).

[7] Stock, J.M., Connelly, J.A., Nowak, M.D., Wenzel, G.W., Redman, K.W., "Optical metrology of the JWST Integrated Science Instrument Module test platform," Proc. SPIE Vol. 7433: Optical System Alignment, Tolerancing, and Verification III, (2009). 